\title{
Association Between Vitamin D and Uric Acid in Adults: A Systematic Review and Meta-Analysis
}

\section{(ㄷ) (i) 우 $\ominus$}

Authors

Ronny Isnuwardana, Sanjeev Bijukchhe, Kunlawat Thadanipon (1), Atiporn Ingsathit, Ammarin Thakkinstian

\author{
Affiliation \\ Department of Clinical Epidemiology and Biostatistics, \\ Faculty of Medicine, Ramathibodi Hospital, Mahidol \\ University, Bangkok, Thailand
}

Key words

vitamin D, uric acid, hyperuricemia, systematic review, meta-analysis

$\begin{array}{ll}\text { received } & 24.02 .2020 \\ \text { accepted after revision } & 07.08 .2020\end{array}$

Bibliography

Horm Metab Res 2020; 52: 732-741

DOI 10.1055/a-1240-5850

ISSN 0018-5043

(c) 2020. The Author(s).

This is an open access article published by Thieme under the terms of the Creative Commons Attribution-NonDerivative-NonCommercial-License, permitting copying and reproduction so long as the original work is given appropriate credit. Contents may not be used for commercial purpose, or adapted, remixed, transformed or built upon. (https://creativecommons.org/licenses/by-nc-nd/4.0/)

Correspondence

Kunlawat Thadanipon

Department of Clinical Epidemiology and Biostatistics

Faculty of Medicine Ramathibodi Hospital

Mahidol University, 270 Rama VI Road

Ratchathewi

10400 Bangkok

Thailand

Tel.: + 662201 1284/Fax: +6622011284

kunlawat.tha@mahidol.ac.th

\author{
$\Theta$ \\ Supplementary material is available under \\ https://doi.org/10.1055/a-1240-5850
}

\begin{abstract}
Association between vitamin D and uric acid is complex and might be bidirectional. Our study aimed to determine the bidirectional association between vitamin $D$ and uric acid in adults. Using MEDLINE via PubMed and Scopus, we systematically searched for observational or interventional studies in adults, which assessed the association between serum vitamin $D$ and serum uric acid, extracted the data, and conducted analysis by direct and network meta-analysis. The present review included 32 studies, of which 21 had vitamin D as outcome and 11 had uric acid as outcome. Meta-analysis showed a significant pooled beta coefficient of serum uric acid level on serum $25(\mathrm{OH}) \mathrm{D}$ level from 3 studies of 0.512 (95\% confidence interval: $0.199,0.825)$ and a significant pooled odds ratio between vitamin D deficiency and hyperuricemia of 1.496 (1.141, $1.963)$. The pooled mean difference of serum $25(\mathrm{OH}) \mathrm{D}$ between groups with hyperuricemia and normouricemia was non-significant at $0.138(-0.430,0.707) \mathrm{ng} / \mathrm{ml}$, and the pooled mean difference of serum uric acid between categories of $25(\mathrm{OH}) \mathrm{D}$ were also non-significant at $0.072(-0.153,0.298)$ $\mathrm{mg} / \mathrm{dl}$ between deficiency and normal, $0.038(-0.216,0.292)$ $\mathrm{mg} / \mathrm{dl}$ between insufficiency and normal, and $0.034(-0.216$, $0.283) \mathrm{mg} / \mathrm{dl}$ between deficiency and insufficiency. In conclusion, increasing serum uric acid might be associated with increasing 25(OH)D level, while vitamin D deficiency is associated with hyperuricemia. These reverse relationships should be further evaluated in a longitudinal study.
\end{abstract}

$\begin{array}{ll}\text { ABBREVIATION } \\ \text { ABCG2 } & \begin{array}{l}\text { Adenosine triphosphate-binding cassette } \\ \text { subfamily G member } 2\end{array} \\ \text { BMI } & \text { Body mass index } \\ \text { CKD } & \text { Chronic kidney disease } \\ \text { CVD } & \text { Cardiovascular disease } \\ \text { eGFR } & \text { Estimated glomerular filtration rate } \\ \text { HT } & \text { Hypertension } \\ \text { MD } & \text { Mean difference } \\ \end{array}$

\begin{tabular}{|c|c|}
\hline NFkB & $\begin{array}{l}\text { Nuclear factor kappa-light-chain-enhancer of } \\
\text { activated B cells }\end{array}$ \\
\hline OR & Odds ratio \\
\hline RoB2 & $\begin{array}{l}\text { Revised Cochrane risk of bias tool for rand- } \\
\text { omized trials }\end{array}$ \\
\hline ROBINS-I & $\begin{array}{l}\text { Risk Of Bias In Non-randomized Studies of } \\
\text { Interventions tool }\end{array}$ \\
\hline SUA & Serum uric acid \\
\hline T2D & Type 2 diabetes \\
\hline VIT D & Vitamin D \\
\hline
\end{tabular}




\section{Introduction}

Vitamin D (VIT D) deficiency is still a major public health problem in the world [1-3], with an estimation of over 1 billion people suffering from VIT D insufficiency or deficiency [4]. VIT D deficiency is not only a problem in areas with limited sunlight exposure such as the polar or temperate regions, but also in the tropics [3]. The prevalence of VIT D insufficiency was reported as 33.5-64.6\% in Thailand [5] and $77.4 \%$ in Brazil [6], similar to the prevalence of $65 \%$ in near-polar Finland [7].

VIT D deficiency could cause bone-related diseases such as rickets and osteoporosis [8] and may increase the risk for cancer [9], tuberculosis [10], and several degenerative diseases such as type 2 diabetes (T2D) [11], hypertension (HT) [12], metabolic syndrome [13], and cardiovascular diseases (CVDs) [14]. VIT D deficiency occurs if there is a decreased intake or synthesis, or increased metabolism or excretion of VIT D. Sources of VIT D are from food and the synthesis by the skin when exposed to sunlight's ultraviolet B ray while it is minimally excreted in a healthy person $[15,16]$. Several factors have been reported to be associated with VIT D level, including age, obesity, several diseases such as chronic kidney disease (CKD), liver disease, tuberculosis, sarcoidosis, and malignancies [17]. In addition, genetic factors may also regulate VIT D, for example, the Fok-I polymorphism of VIT D receptor gene [18] and the cytochrome P450 24A1 [19] and 2R1 [20-22] genes.

Previous evidence [23-27] showed that VIT D deficiency might increase the risk of high serum uric acid (SUA). On the other hand, some studies [28-32] showed that high SUA (hyperuricemia) could decrease VIT D level and/or induce VIT D deficiency. Therefore, we conducted a systematic review and meta-analysis to assess whether VIT D deficiency is associated with high SUA or vice versa.

\section{Materials and Methods}

The study was conducted after registering with PROSPERO (CRD42 018105283). It has no funding source. A literature search was performed in MEDLINE via PubMed and Scopus databases to identify relevant studies published through July 31st, 2018. The search strategy was provided in Supplementary Tables 15 and 25 .

\section{Selection of studies}

Studies were included if they were comparative studies, which assessed the association between serum VIT D and SUA in adults. They were excluded if they were published in untranslatable languages, multiple publications of the same original research, or had incomplete data after 3 attempts of contacting the authors.

\section{Study factor and outcome of interest}

The main exposure and outcome were VIT D and SUA level which were measured by any method according to the original studies. The SUA could be dealt with as a continuous variable or categorized using different cut-off points (i.e., $5.7,6$, or $6.6 \mathrm{mg} / \mathrm{dl}$ in females and 7 or $7.7 \mathrm{mg} / \mathrm{dl}$ in males). VIT D level could be measured as calcifediol $[25(\mathrm{OH}) \mathrm{D}]$ and calcitriol $\left[1,25(\mathrm{OH})_{2} \mathrm{D}\right]$ forms. The $25(\mathrm{OH})$ $D$ level was categorized into deficiency, insufficiency, and normal if it was $<20,20-30$, and $>30 \mathrm{ng} / \mathrm{ml}$, respectively.

\section{Data extraction}

Data extraction was done using a standardized data extraction form independently by 2 reviewers (RI and SB) and checked by senior reviewers (KT and AT). The data extraction form included characteristics of the article, study, and participants, exposure and outcome data of VIT D and SUA, and covariables, including mean age, body mass index (BMI), serum creatinine, serum glycated hemoglobin (HbA1c) levels, estimated glomerular filtration rate (eGFR), percentage of males, obesity, smoking, T2D, HT, CKD, and CVD. All disagreements were solved by discussion and reference to the original article with consent from a senior reviewer (KT).

\section{Risk of bias assessment}

Two reviewers (RI and SB) assessed the risk of bias for each study independently. For observational studies, we used the Newcastle Ottawa Scale for cohort [33], adapted for cross-sectional [34] and case-control [33] studies, while the revised Cochrane risk of bias tool for randomized trials (RoB2) [35] and Risk Of Bias In Non-randomized Studies of Interventions tool (ROBINS-I) [36] were used for interventional studies. All disagreements were solved by discussion between the 2 reviewers (RI and SB) with consent from a senior reviewer (KT).

\section{Statistical analysis}

Direct meta-analysis

Direct meta-analysis was performed to pool the effect sizes, including beta coefficients (slopes) of SUA level on serum VIT D $(25(\mathrm{OH})$ D) level, odds ratio (OR) between hyperuricemia and VIT D deficiency, and unstandardized mean difference (MD) of serum VIT D between the subjects with hyperuricemia and normal SUA. Heterogeneity was assessed and it was considered present if a Cochrane $Q$ test $p$-value was $<0.1$ or Higgins $I^{2} \geq 25 \%$ [37]. Effect sizes were pooled using DerSimonian and Laird method if they were heterogeneous, otherwise, the inverse-variance method was used [37].

We explored the source of heterogeneity using Galbraith plot and sensitivity or subgroup analysis where appropriate. A potential source (e.g., T2D, CVD, HT, CKD, age, BMI, and obesity) was fitted individually in a meta-regression model. If the $\mathrm{T}^{2}$ was decreased by $\geq 50 \%$, a subgroup analysis was performed accordingly [38]. Publication bias was assessed by funnel plot and Egger test [39].

\section{Network meta-analysis}

Network meta-analysis using two-stage approach was applied to estimate MDs of SUA between groups with different VIT D status (i. e., normal, insufficiency, and deficiency). Initially, linear regression analysis was applied to estimate the MD along with the variance-covariance for each individual study using normal VIT D status as the reference group. Then, a multivariate random-effects meta-analysis with a consistency model was used to pool the MDs across the studies $[40,41]$.

Inconsistency was assessed using the design-by-treatment interaction model [42]. Publication bias was assessed using a comparison-adjusted funnel plot [43].

All statistical analysis was performed using Stata version 15.1 SE by StataCorp (College Station, Texas, USA). A p-value < 0.05 was 
the threshold for statistical significance, except for heterogeneity where $\mathrm{p}$-value $<0.1$ was used.

\section{Results}

\section{Study Selection}

There were 243 and 804 articles identified from the MEDLINE and Scopus databases, respectively. Thirty-two studies met our eligibility criteria (see Supplementary $>$ Fig. 1S). Characteristics of the studies are described in $>$ Table 1. Most of them (23) were crosssectional studies. Their participants were either from the general population or had specific diseases, with mean age ranging from 36.9 to 76.9 years.

Among 32 studies, 21 [28-32, 44-59] and 11 [23-27, 60-65] considered VIT D and SUA as the outcome, respectively. Within the 21 studies with VIT D outcome, $6[31,32,44,48-50]$ provided data of SUA and VIT D levels as continuous variables, 14 [28-32, 45$47,49,52-54,57,59]$ assessed association between SUA level as continuous data and categorical VIT D status, while $3[55,56,58]$ provided categorical data for both. For the 11 studies with SUA outcome, 1 [64] had continuous data for both VIT D and SUA levels, $9[23-25,27,60-63,65]$ provided continuous data for VIT D level and categorical SUA status, and $2[25,26]$ provided categorical data for both.

\section{Risk of bias assessment}

The risk of bias assessment was performed and the results can be seen in Supplementary Table 3S-5S. For observational studies, 20 [23-27, 29, 30, 32, 45-47, 53, 57, 59-63, 65, 66], 4 [51, 52, 56, 58], and $6[28,44,50,54,55,64]$ were of low, moderate, and high risk of bias, respectively. The 2 interventional studies $[31,48]$ included had low risk of bias.

\section{Effects of SUA on VIT D outcome}

Beta coefficient

Three studies $[31,32,48]$ reported beta coefficient of SUA level on serum 25(OH)D level (see $>$ Fig. 1 and Supplementary Table 6S) with the pooled coefficient ( $95 \%$ confidence interval) of 0.512 $\left(0.199,0.825 ; I^{2}=8.1 \%\right)$. In other words, there was a statistically significant, positive correlation between SUA and VIT D levels in that each $\mathrm{mg} / \mathrm{dl}$ increase in SUA level was associated with an increase in $25(\mathrm{OH}) \mathrm{D}$ by $0.512 \mathrm{ng} / \mathrm{ml}$. The funnel plot was symmetrical, corresponding to the non-significant Egger test (coefficient $=1.94$, standard error $=2.19$, $\mathrm{p}$-value $=0.539$ ).

\section{Mean difference in SUA}

Thirteen studies assessed associations between SUA and VIT D. Among them, 9 studies [29-32, 45-47, 49, 59] compared SUA levels between VIT D deficiency, insufficiency, and normal groups, whereas $4[28,53,54,57]$ reported SUA only between deficiency or insufficiency and normal groups. The most commonly used cutoffs for deficiency and insufficiency were $<20$ and $<30 \mathrm{ng} / \mathrm{ml}$, respectively (see Supplementary Table 7S). A network meta-analysis applied to estimate MDs of SUA levels among 3 groups across the studies yielded the non-significant pooled MDs of 0.072
$(-0.153,0.298), 0.038(-0.216,0.292)$, and $0.034(-0.216$, $0.283) \mathrm{mg} / \mathrm{dl}$ for deficiency vs normal, insufficiency vs normal, and deficiency vs insufficiency, respectively; all of them were not significant (see $>$ Fig. 2 and Table 8S). Comparison-adjusted funnel plot from the network meta-analysis was symmetrical, indicating no significant publication bias.

Exploring the source of heterogeneity by subgroup analysis in the first direct meta-analysis (VIT D deficiency vs. normal) based on percentage of T2D patients showed that studies with partial T2D patients $[29,45]$ had a statistically significant pooled MD in SUA level of $-0.379 \mathrm{mg} / \mathrm{dl}\left(-0.552,-0.205 ; \mathrm{I}^{2}=0.0 \%\right)$, compared to the non-significant pooled MD of $-0.247 \mathrm{mg} / \mathrm{dl}(-0.874,0.379$; $\mathrm{I}^{2}=74.9 \%$ ) from studies with $100 \%$ T2D patients [ 28,57$]$ (see Supplementary Tables 95). From the second direct meta-analysis (insufficiency vs normal), subgroup analysis by BMI showed that the studies with mean $\mathrm{BMI}<30 \mathrm{~kg} / \mathrm{m}^{2}[29,49]$ had a non-significant pooled MD of $0.087 \mathrm{mg} / \mathrm{dl}\left(-0.306,0.132 ; \mathrm{I}^{2}=0.0 \%\right)$ and those with higher mean BMI [45-47, 59] showed a non-significant pooled MD of $0.076 \mathrm{mg} / \mathrm{dl}\left(-0.240,0.393 ; \mathrm{I}^{2}=58.8 \%\right.$ ) (see Table 10S). No specific source of heterogeneity was found in the direct meta-analysis of deficiency vs insufficiency groups.

\section{Effects of VIT D on SUA outcome}

\section{Odds ratio}

Five studies $[25,26,55,56,58]$ assessed association between VIT $D$ deficiency and hyperuricemia (see Table 11S). ORs were estimated and pooled across studies, yielding the statistically significant pooled OR of $1.496\left(1.141,1.963 ;\left.\right|^{2}=3.5 \%\right)$, indicating a significant association in which the odds for hyperuricemia of the group with VIT D deficiency was 1.496 times that of the group with normal VIT D. There is no evidence of publication bias by the funnel plot (see $>$ Fig. 3 ) and Egger test (coefficient $=-0.188$; standard error $=0.593 ; p$-value $=0.772$ ).

\section{Mean difference of VIT D}

Eight studies [23-25, 27, 60-62, 65] compared 25(OH)D levels between hyperuricemia and normouricemia groups (see Table 12S). The MDs were moderately heterogeneous $\left(I^{2}=30.0 \%\right)$ with the non-significant pooled MD of $0.138 \mathrm{ng} / \mathrm{ml}(-0.430,0.707$; see - Fig. 4). The funnel plot and Egger test showed no evidence of publication bias (coefficient $=-0.726$; standard error $=1.162$; $\mathrm{p}$-value $=0.555)$.

\section{Discussion}

We performed a systematic review and meta-analysis on the association between serum VIT D level and SUA level and vice versa, pooling the effect sizes of association between VIT D and SUA. We found that each $\mathrm{mg} / \mathrm{dl}$ increase in SUA corresponded to an increase in serum $25(\mathrm{OH}) \mathrm{D}$ by approximately $0.5 \mathrm{ng} / \mathrm{ml}$. Additionally, the odds for hyperuricemia of VIT D deficiency was approximately 1.5 times that of normal VIT D level.

According to our findings, high SUA might increase 25(OH)D level. This may be explained by the fact that hyperuricemia can inhibit $1 \alpha$-hydroxylase and prevent the conversion of the $25(\mathrm{OH}) \mathrm{D}$ form of VIT D into calcitriol $\left[1,25(\mathrm{OH})_{2} \mathrm{D}\right][25,66]$, hence increasing the level of 25(OH)D but decreasing the level of calcitriol. Ge- 


\begin{tabular}{|c|c|c|c|c|c|c|c|c|c|c|c|c|c|c|c|c|c|c|}
\hline ฏ̊̊ & $\stackrel{\circ}{\circ}$ & & & & & & & & & $\begin{array}{l}\stackrel{a}{\dot{n}} \\
\text { nes }\end{array}$ & 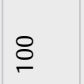 & & & & & & & \\
\hline ฏ̊ & 0 & & & & & & & & & 㤩 & & & & & & 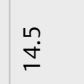 & & \\
\hline ఏ̊ำ & $\overline{i n}$ & & & & & & & $\overline{\dot{\sigma}}$ & & Wె & $\underset{\infty}{+}$ & & & & & 竓 & & \\
\hline ఏे̊ & 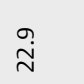 & & & & & & & $\stackrel{8}{\circ}$ & & & $\frac{\stackrel{\llcorner}{x}}{\dot{\gamma}}$ & & & & & $\begin{array}{l}\dot{\varphi} \\
\stackrel{\dot{\varphi}}{L}\end{array}$ & & \\
\hline 芒 & & & & & $\begin{array}{l}\stackrel{\stackrel{\leftrightarrow}{\sim}}{\sim} \\
\end{array}$ & & & $\underset{\sim}{\stackrel{n}{\sim}}$ & & $\bar{g}$ & & & $\hat{\grave{\omega}}$ & & & mे. & & \\
\hline ةัँ & & & & & & & $\stackrel{\infty}{\overleftrightarrow{\sigma}}$ & & & & & & & & & & & \\
\hline 离 & $\bar{i}$ & $\stackrel{\stackrel{\leftrightarrow}{\longrightarrow}}{\longrightarrow}$ & 点 & นึ่ & 0 & $\begin{array}{l}\stackrel{\mathscr{m}}{\mathrm{m}} \\
\end{array}$ & $\stackrel{m}{\stackrel{m}{n}}$ & ்ָ & $\begin{array}{l}\text { Ln? } \\
\stackrel{\infty}{\sigma}\end{array}$ & $\stackrel{\text { n̊ }}{R}$ & di & $\begin{array}{l}\infty \\
\infty \\
\infty\end{array}$ & $\dot{J}$ & 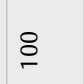 & $\approx$ & 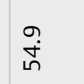 & 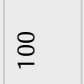 & 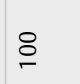 \\
\hline 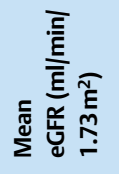 & & & & & $\begin{array}{l}\text { L̊n } \\
\text { ஸे } \\
\infty\end{array}$ & $\hat{\tilde{\sigma}}$ & & & & & $\begin{array}{l}\text { సે } \\
\stackrel{N}{N}\end{array}$ & $\begin{array}{l}\hat{m} \\
\infty \\
\infty\end{array}$ & & & & & & $\begin{array}{l}\text { के } \\
\text { ¿ }\end{array}$ \\
\hline 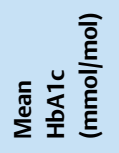 & & $\stackrel{\substack{\infty \\
\triangleright}}{ }$ & 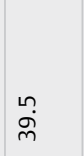 & & & ָิ & & $\frac{m}{\infty}$ & 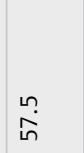 & $\begin{array}{l}\stackrel{\text { L }}{+} \\
\stackrel{5}{n}\end{array}$ & & & & & & & & \\
\hline 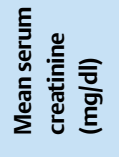 & gे & : & g. & & & $\stackrel{\infty}{0}$ & & & $\begin{array}{l}\stackrel{0}{\infty} \\
0 \\
0\end{array}$ & 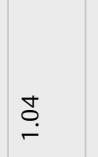 & & 令 & & $\begin{array}{c}\stackrel{L}{\infty} \\
\stackrel{0}{\circ}\end{array}$ & & & ô. & $\stackrel{\mathscr{L}}{-}$ \\
\hline 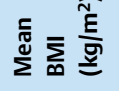 & $\stackrel{\sim}{\stackrel{\infty}{\sim}}$ & $\begin{array}{l}0 \\
\stackrel{N}{N}\end{array}$ & & & i̊. & & & 亡ّ & 迥 & 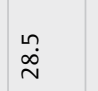 & $\underset{\sim}{\stackrel{\sim}{\sim}}$ & & & $\ddot{\dot{w}}$ & & $\stackrel{m}{\sim}$ & 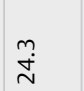 & হి \\
\hline 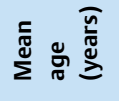 & กิ & $\ddot{\gamma}$ & $\stackrel{t}{8}$ & $\begin{array}{l}\infty \\
\dot{m} \\
\dot{m}\end{array}$ & 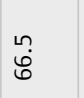 & $\stackrel{n}{n}{ }_{n}^{n}$ & $\overline{\dot{g}}$ & ત̀. & $\begin{array}{l}\dot{\infty} \\
\stackrel{\infty}{\sigma}\end{array}$ & $\overline{8}$ & $\begin{array}{l}\sigma \\
\infty \\
0\end{array}$ & 文 & $\begin{array}{l}\infty \\
\stackrel{\infty}{0} \\
\stackrel{h}{n}\end{array}$ & ن́ & $\overline{\vec{m}}$ & Õ & 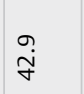 & ڤి \\
\hline 岌 气̆ & 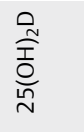 & 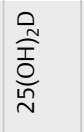 & 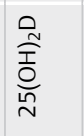 & 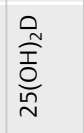 & $\begin{array}{l}\stackrel{\overline{0}}{\bar{v}} \\
\stackrel{\breve{5}}{5}\end{array}$ & 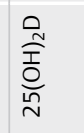 & 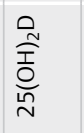 & 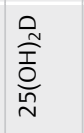 & 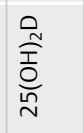 & 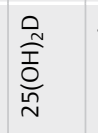 & $\begin{array}{l}\stackrel{\bar{J}}{\widetilde{J}} \\
\stackrel{\breve{S}}{5}\end{array}$ & 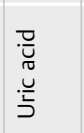 & 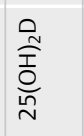 & 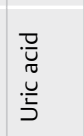 & 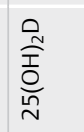 & 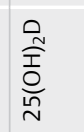 & 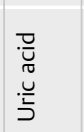 & 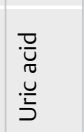 \\
\hline 㝘 & 离 & 趈 & $\breve{s}$ & 胥 & 王 & 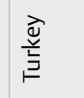 & $\begin{array}{l}\frac{0}{5} \\
\frac{\sqrt{5}}{5} \\
\frac{5}{5}\end{array}$ & 胥 & $\begin{array}{l}\text { 营 } \\
\text { 员 }\end{array}$ & $\begin{array}{l}\frac{\lambda}{\bar{I}} \\
\end{array}$ & 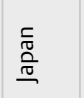 & 氞 & 莺 & 苞 & $\begin{array}{l}\frac{0}{5} \\
\vdots \\
3\end{array}$ & 疍 & $\frac{\frac{i}{\pi}}{\sum^{\pi}} \cdot \frac{\pi}{n}$ & $\begin{array}{l}\text { 듬 } \\
\text { กิ }\end{array}$ \\
\hline 点 & 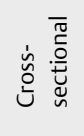 & 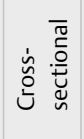 & 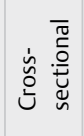 & 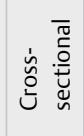 & 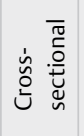 & 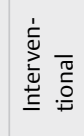 & 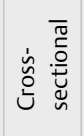 & 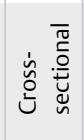 & 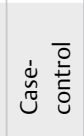 & 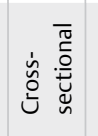 & 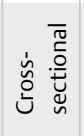 & 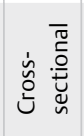 & 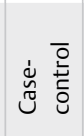 & 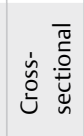 & 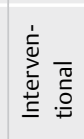 & $\begin{array}{l}\text { to } \\
\text { 흥 }\end{array}$ & 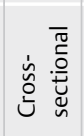 & 岕 \\
\hline 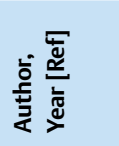 & 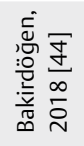 & 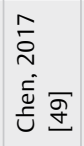 & 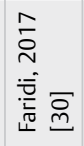 & 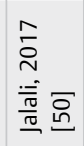 & 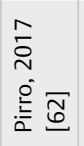 & 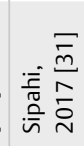 & 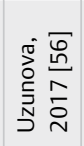 & 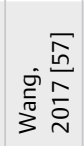 & 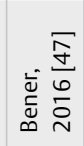 & 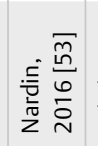 & 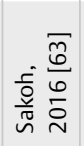 & 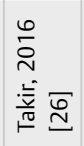 & 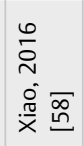 & 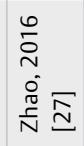 & 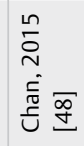 & 它 & 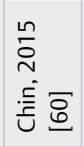 & 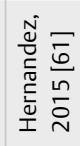 \\
\hline
\end{tabular}




\begin{tabular}{|c|c|c|c|c|c|c|c|c|c|c|c|c|c|c|}
\hline ڤ̊ํ & & & & & & & & & & & & & ঃ & \\
\hline 仓े & & & 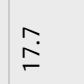 & & & & & & & & $\overline{\dot{\sim}}$ & & & \\
\hline 总 & & & & & & & $\begin{array}{l}\stackrel{n}{n} \\
\stackrel{\varphi}{\varphi}\end{array}$ & $\stackrel{\infty}{\dot{q}}$ & & & $\begin{array}{l}\dot{0} \\
\text { जे }\end{array}$ & & & \\
\hline ڤ̊ํ & & & $\check{\check{n}}$ & & & $\begin{array}{l}\dot{0} \\
\infty \\
\infty\end{array}$ & 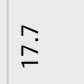 & $\stackrel{\sim}{\sim}$ & & & 0 & & & 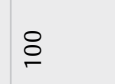 \\
\hline 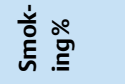 & & & $\begin{array}{l}\infty \\
\grave{\sim} \\
\stackrel{\infty}{~}\end{array}$ & & & $\begin{array}{l}\stackrel{\sim}{0} \\
\infty \\
\leftarrow\end{array}$ & & $\stackrel{\bullet}{\circ}$ & & f & $\underset{\mathrm{m}}{\stackrel{\sim}{i}}$ & $\widehat{\grave{o}}$ & & \\
\hline $\begin{array}{l}\text { すั } \\
\text { ठิ }\end{array}$ & & & & & & & 官 & & & $\stackrel{\infty}{\stackrel{\infty}{n}}$ & & & & \\
\hline 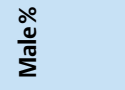 & 岗 & $\stackrel{n}{\kappa}$ & జే & $\begin{array}{l}\stackrel{\bullet}{\dot{J}} \\
\text {. }\end{array}$ & 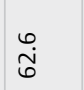 & 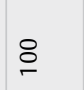 & g̊ & 0 & $\underset{\substack{n \\
n}}{ }$ & ণ্் & 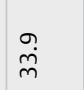 & 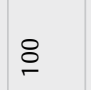 & $\stackrel{\mathscr{Y}}{\mathscr{\gamma}}$ & F \\
\hline 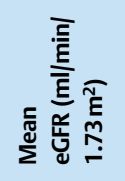 & & & $\begin{array}{l}\stackrel{f}{0} \\
\stackrel{2}{R}\end{array}$ & & & $\begin{array}{l}\infty \\
\stackrel{+}{+} \\
\end{array}$ & & & & $\begin{array}{l}\text { Бे } \\
\text { ம் }\end{array}$ & & $\hat{o}_{0}^{\circ}$ & & 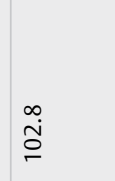 \\
\hline 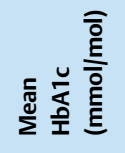 & & & & & & & & & & & 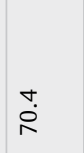 & & & 宊 \\
\hline 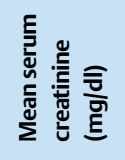 & & $\stackrel{9}{\rightleftharpoons}$ & & & & 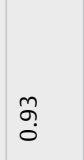 & शे. & & & & 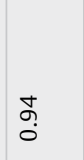 & $\stackrel{\infty}{\stackrel{0}{-}}$ & $\frac{m}{m}$ & g. \\
\hline$\sum_{\Sigma}^{\bar{D}} \sum_{\infty} \frac{\widetilde{\widetilde{E}}}{\bar{E}}$ & & & $\stackrel{9}{\stackrel{2}{\sim}}$ & 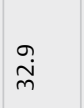 & $\stackrel{\stackrel{n}{\sim}}{\sim}$ & 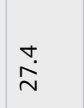 & $\frac{m}{m}$ & $\stackrel{\stackrel{J}{d}}{\sim}$ & & & $\frac{m}{m}$ & $\stackrel{\infty}{\stackrel{\infty}{\sim}}$ & $\hat{\text { ஸे }}$ & $\stackrel{m}{\stackrel{m}{\sim}}$ \\
\hline 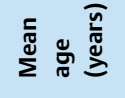 & 节 & : & 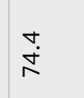 & $\begin{array}{l}b_{0}^{\circ} \\
\dot{\sigma}\end{array}$ & $\begin{array}{l}\infty \\
\dot{y} \\
\dot{q}\end{array}$ & $\stackrel{\infty}{\stackrel{\infty}{r}}$ & $\stackrel{m}{n}$ & & & $\begin{array}{l}\stackrel{\vartheta}{\dot{y}} \\
\dot{q}\end{array}$ & 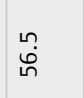 & g̊ & 館 & 㸳 \\
\hline ذُ & 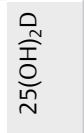 & 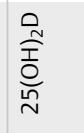 & 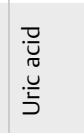 & 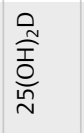 & 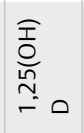 & 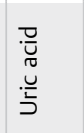 & 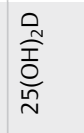 & 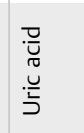 & 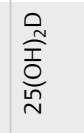 & 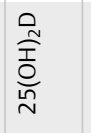 & 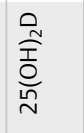 & 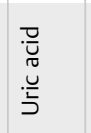 & 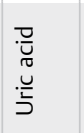 & 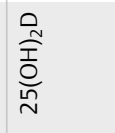 \\
\hline 言 & $\begin{array}{l}\frac{7}{5} \\
\frac{\pi}{0} \\
\end{array}$ & 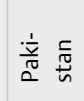 & $\frac{\text { त्र }}{\underline{\underline{N}}}$ & 吕 & 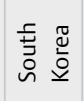 & 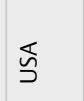 & $\begin{array}{l}\frac{\cdot}{\bar{\pi}} \\
\text { जิ }\end{array}$ & 苞 & $\begin{array}{l}\overline{\overline{0}} \\
\overline{\tilde{a}}\end{array}$ & 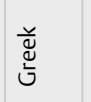 & 离 & 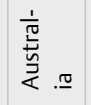 & 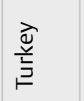 & 疍 \\
\hline 喜 & $\begin{array}{l}\text { t. } \\
\text { 흥 }\end{array}$ & 离 & 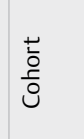 & 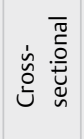 & 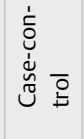 & 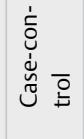 & 空 & 尊 & 峞 & 岕离 & 离 & 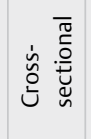 & 岕 & 崖 \\
\hline 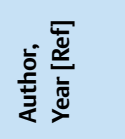 & 离 & 离 & 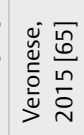 & 递 & 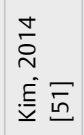 & 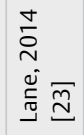 & 迎 & 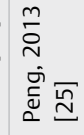 & 苞怘 & 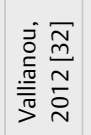 & 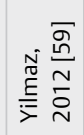 & 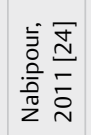 & 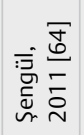 & 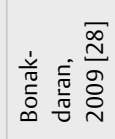 \\
\hline
\end{tabular}


a

Association in slope coefficients between SUA and VIT D level

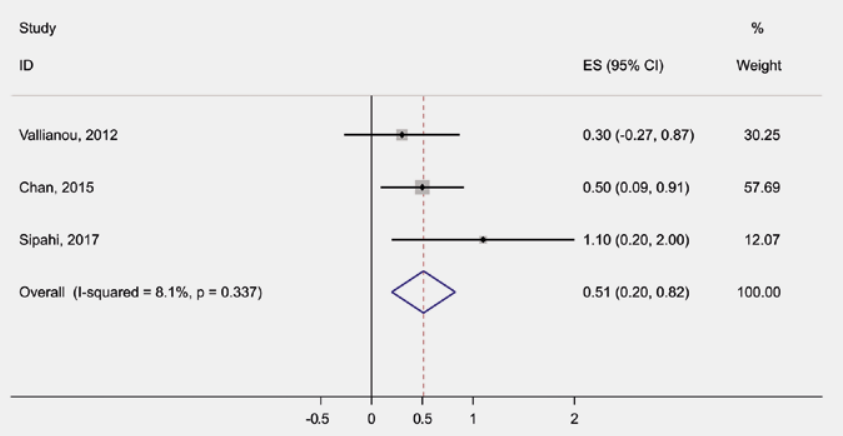

b

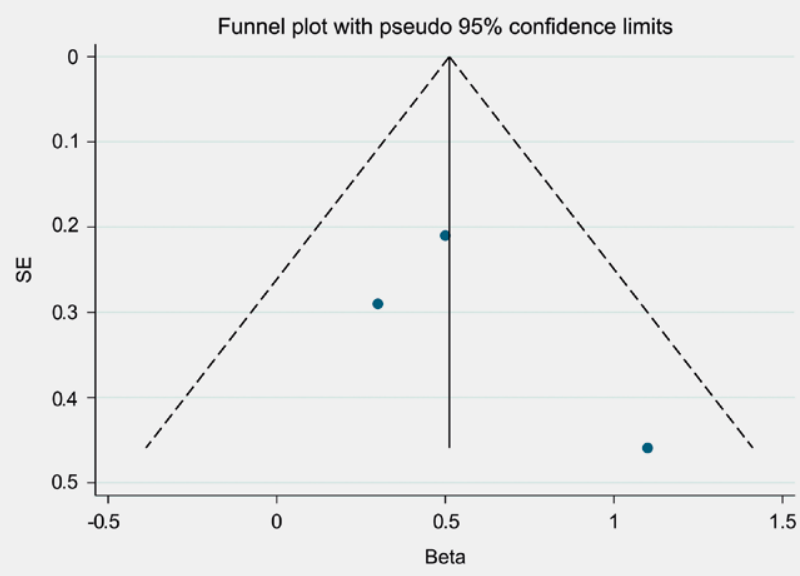

- Fig. 1 Meta-analysis of beta coefficients of SUA level on serum VIT D level: a forest plot and $\mathbf{b}$ funnel plot.

a

$$
\text { Mean difference of SUA (mg/dl) }
$$$$
\text { in VIT D deficiency group vs normal }
$$

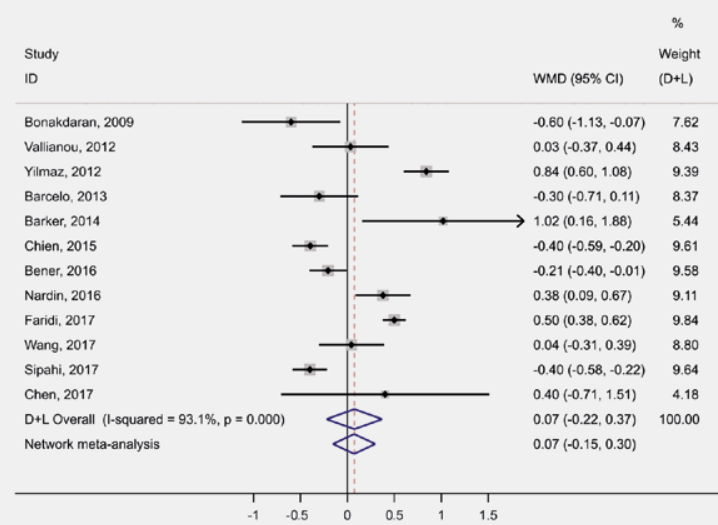

c

Mean difference of SUA (mg/dl)

in VIT D deficiency group vs insufficiency

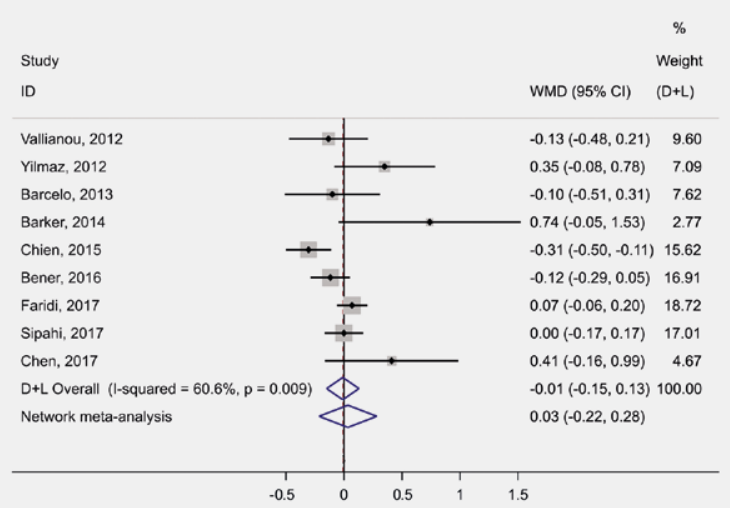

b

\section{Mean difference of SUA (mg/dl)}

in VIT D insufficiency group vs normal

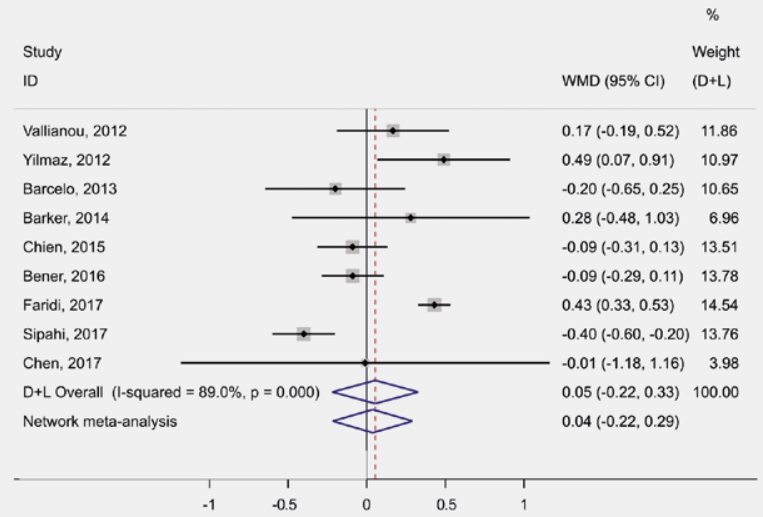

d

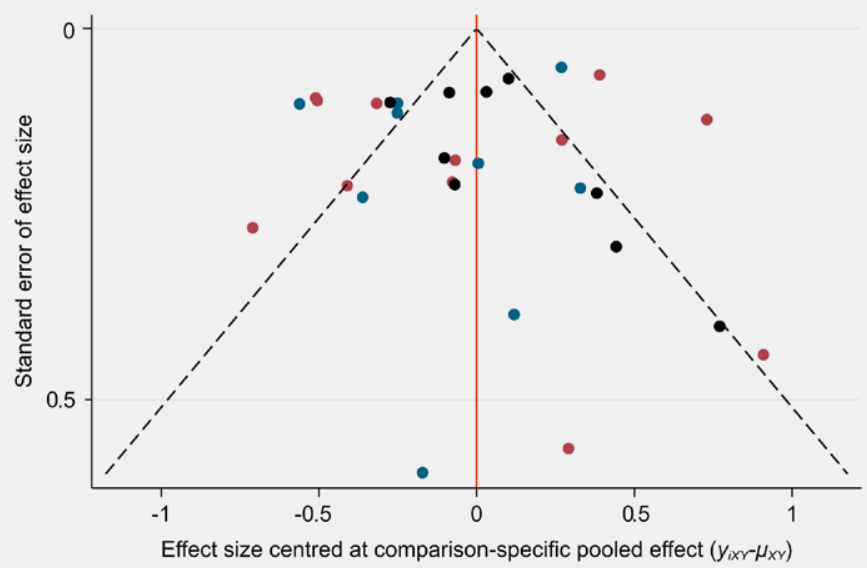

- Avs B $\bullet$ Avs C $\bullet$ B vs C

-Fig. 2 Meta-analysis of mean differences in SUA levels between categories of VIT D status: forest plots for a deficiency vs. normal, b insufficiency vs. normal, (c deficiency vs. insufficiency, and $\mathbf{d}$ comparison-adjusted funnel plot from network meta-analysis. 
a

Odds ratio of vitamin $D$ deficiency in hyperuricemia group vs normal

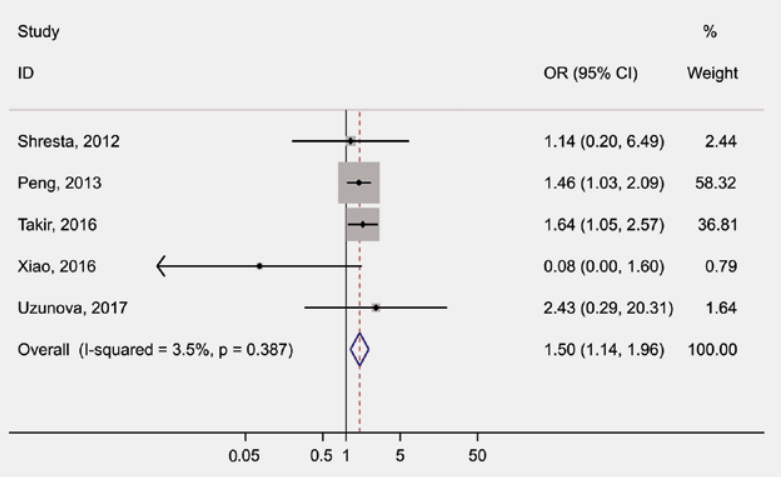

b

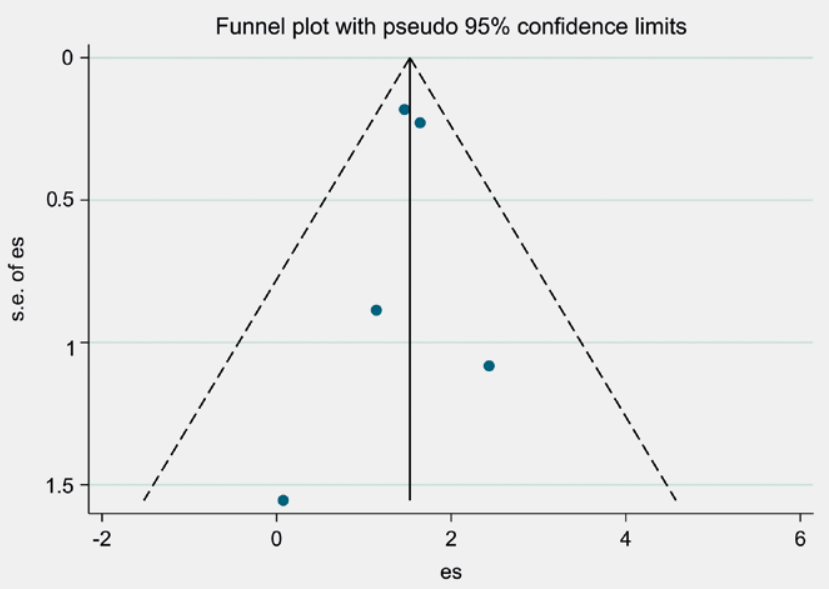

-Fig. 3 Meta-analysis of odds ratios between VIT D deficiency and hyperuricemia: a forest plot and $\mathbf{b}$ funnel plot. Mean difference of VIT D ( $\mathrm{ng} / \mathrm{ml})$ in hyperuricemia group vs normal

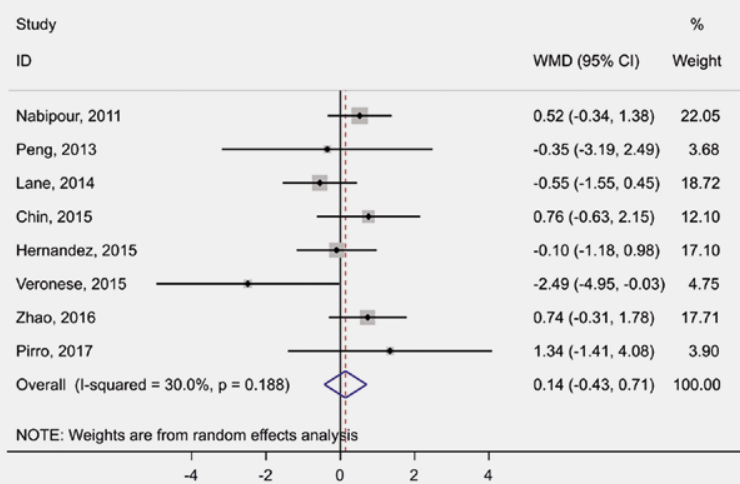

b

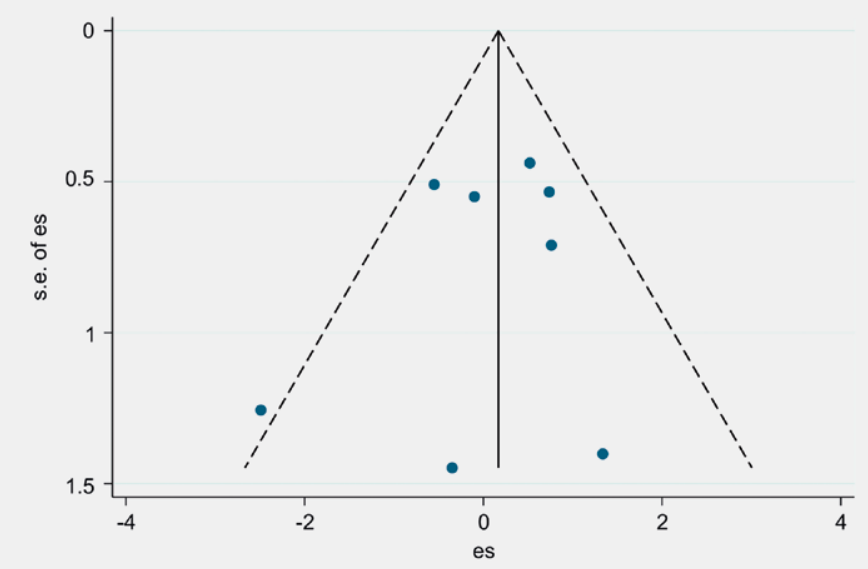

-Fig. 4 Meta-analysis of mean differences in serum VIT D levels between groups with hyperuricemia and normal SUA levels. a forest plot and b funnel plot.

netic backgrounds may also be involved in these metabolic pathways as shown by evidence from genome-wide association studies [67-71]. For instance, the rs4588 and rs2282679 polymorphisms of GC (VIT D binding protein) gene and rs 10766197 polymorphism of cytochrome P450 2R1 gene were highly associated with VIT D level $[72,73]$, whereas the rs 2231142 polymorphism of adenosine triphosphate-binding cassette subfamily $G$ member 2 (ABCG2) gene was associated with increased SUA level [71]. A Mendelian randomization study [74] also showed a causal association between the rs2231142 polymorphism of ABCG2 gene and VIT D through SUA by reducing urate transporter in renal proximal tubules. In the same site, the increasing SUA is associated with an inhibition of CYP27B1 gene expression for the $1 \alpha$-hydroxylase enzyme, which converts 25(OH)D to calcitriol [66]. Furthermore, increased level of SUA might also lead to nephropathy in diabetic patients or initi- ation and progression of renal disease in non-diabetic patients, manifested as microalbuminuria due to hyperuricemia [75], which might reduce the circulating VIT D binding protein and decrease VIT D level. Conversely, treatment with allopurinol, a xanthine oxidase inhibitor, in diabetic patients can decrease the SUA level and also increase the serum vitamin D level [76]. Therefore, the direction of the relationship between SUA and VIT D levels is still controversial.

It should be noted also that the study with the biggest weight for the pooled beta coefficient was a study using worldwide data on patients with chronic hepatitis B [48], which could, in turn, cause detrimental effects on serum VIT D level. However, this description might only explain a part of the bigger picture, since there are several other factors which could affect VIT D level such as diet, BMI, age, gender, geographical location, and season [21]. 
Meanwhile, for the association between VIT D and SUA outcome, the results from pooling ORs $[25,26,55,56,58]$ showed a significant association between VIT D deficiency and hyperuricemia. This pooled OR is inconsistent with the above-mentioned pooled beta coefficient of SUA on serum 25(OH)D level. The mechanism behind this might be the secondary hyperparathyroidism caused by VIT D deficiency [77] which affects the ABCG2 gene [78] and thus decreases excretion or increases reabsorption of uric acid in the kidney tubules [60]. This VIT D deficiency could be caused by limited intake, insufficient ultraviolet exposure from sunlight, or genetic factors. It should be noted that as genetic factors can affect both VIT D and SUA levels as previously mentioned, each with their own specific gene polymorphisms, the fact that these genetic factors are not evenly distributed across different populations might also explain the conflicting results for the associations between SUA and VIT D, being pooled from a number of different populations.

This study is the first systematic review on the bidirectional association between SUA and VIT D which includes a total of 32 studies within the analysis. However, there are also some limitations in the present review, for example, the 2 significant pooled effect sizes are from a limited number of studies, whereas the other meta-analyses which consist of higher number of studies produce heterogeneous results.

In summary, increasing SUA might increase 25(OH)D VIT D level, while VIT D deficiency is associated with hyperuricemia. These reverse relationships should be further evaluated in a longitudinal study.

\section{Conflict of Interest}

The authors declare that they have no conflict of interest.

\section{References}

[1] Hossein-nezhad A, Holick MF. Vitamin D for health: A global perspective. Mayo Clin Proc 2013; 88: 720-755

[2] Mithal A, Wahl DA, Bonjour JP et al. Global vitamin D status and determinants of hypovitaminosis D. Osteoporos Int 2009; 20: 1807-1820

[3] Palacios C, Gonzalez L. Is vitamin D deficiency a major global public health problem? J Steroid Biochem Mol Biol 2014; 144 Pt A: 138-145

[4] Holick MF, Chen TC. Vitamin D deficiency: A worldwide problem with health consequences. Am J Clin Nutr 2008; 87: 1080s-1086s

[5] Chailurkit LO, Aekplakorn W, Ongphiphadhanakul B. Regional variation and determinants of vitamin $D$ status in sunshine-abundant Thailand. BMC Public Health 2011; 11: 853

[6] Unger MD, Cuppari L, Titan SM et al. Vitamin D status in a sunny country: where has the sun gone? Clin Nutr 2010; 29: 784-788

[7] Virtanen JK, Nurmi T, Voutilainen $S$ et al. Association of serum 25-hydroxyvitamin $\mathrm{D}$ with the risk of death in a general older population in Finland. Eur J Nutr 2011; 50: 305-312

[8] Cranney A, Horsley T, O'Donnell $\mathrm{S}$ et al. Effectiveness and safety of vitamin $D$ in relation to bone health. Evid Rep Technol Assess (Full Rep) 2007; 158: 1-235

[9] Feldman D, Krishnan AV, Swami S et al. The role of vitamin D in reducing cancer risk and progression. Nat Rev Cancer 2014; 14: 342-357
[10] Facchini L, Venturini E, Galli L et al. Vitamin D and tuberculosis: A review on a hot topic. J Chemother 2015; 27: 128-138

[11] Mitri J, Muraru MD, Pittas AG. Vitamin D and type 2 diabetes: A systematic review. Eur J Clin Nutr 2011; 65: 1005-1015

[12] Vimaleswaran KS, Cavadino A, Berry DJ et al. Association of vitamin D status with arterial blood pressure and hypertension risk: A mendelian randomisation study. Lancet Diabetes Endocrinol 2014; 2: 719-729

[13] Huang CY, Chang HH, Lu CW et al. Vitamin D status and risk of metabolic syndrome among non-diabetic young adults. Clin Nutr 2015; 34: 484-489

[14] Norman PE, Powell JT. Vitamin D and cardiovascular disease. Circ Res 2014; 114: 379-393

[15] Saraff V, Shaw N. Sunshine and vitamin D. Arch Dis Child 2016; 101: 190-192

[16] Thrailkill KM, Jo CH, Cockrell GE et al. Enhanced excretion of vitamin D binding protein in type 1 diabetes: a role in vitamin $D$ deficiency? J Clin Endocrinol Metab 2011; 96: 142-149

[17] Tsiaras WG, Weinstock MA. Factors influencing vitamin D status. Acta Derm Venereol 2011; 91: 115-124

[18] Agnello L, Scazzone C, Ragonese P et al. Vitamin D receptor polymorphisms and 25-hydroxyvitamin $D$ in a group of Sicilian multiple sclerosis patients. Neurol Sci 2016; 37: 261-267

[19] Agnello L, Scazzone C, Lo Sasso B et al. CYP27A1, CYP24A1, and RXR-a Polymorphisms, Vitamin D, and Multiple Sclerosis: a Pilot Study. J Mol Neurosci 2018; 66: 77-84

[20] Scazzone C, Agnello L, Ragonese P et al. Association of CYP2R1 rs10766197 with MS risk and disease progression. J Neurosci Res 2018; 96: 297-304

[21] Fohner AE, Wang Z, Yracheta J et al. Genetics, diet, and season are associated with serum 25-hydroxycholecalciferol concentration in a Yup'ik Study Population from Southwestern Alaska. J Nutr 2016; 146: 318-325

[22] Duan L, Xue Z, Ji H et al. Effects of CYP2R1 gene variants on vitamin D levels and status: A systematic review and meta-analysis. Gene 2018; 678: 361-369

[23] Lane NE, Parimi N, Lui LY et al. Association of serum uric acid and incident nonspine fractures in elderly men: The Osteoporotic Fractures in Men (MrOS) study. J Bone Miner Res 2014; 29: 1701-1707

[24] Nabipour I, Sambrook PN, Blyth FM et al. Serum uric acid is associated with bone health in older men: A cross-sectional population-based study. J Bone Miner Res 2011; 26: 955-964

[25] Peng H, Li H, Li C et al. Association between vitamin D insufficiency and elevated serum uric acid among middle-aged and elderly Chinese Han women. PLoS One 2013; 8: e61159

[26] Takir M, Solak Y, Erek A et al. Association between elevated serum uric acid and vitamin $D$ insufficiency among the middle-aged and elderly population. Turk Nephrol Dial Transplant J 2016; 25: 182-186

[27] Zhao DD, jiao PL, Yu J] et al. Higher serum uric acid is associated with higher bone mineral density in Chinese men with type 2 diabetes mellitus. Int J Endocrinol 2016; 2528956

[28] Bonakdaran S, Varasteh AR. Correlation between serum 25 hydroxy vitamin D3 and laboratory risk markers of cardiovascular diseases in type 2 diabetic patients. Saudi Med J 2009; 30: 509-514

[29] Chien KL, Hsu HC, Chen PC et al. Total 25-hydroxyvitamin D concentration as a predictor for all-cause death and cardiovascular event risk among ethnic Chinese adults: A cohort study in a Taiwan community. PLoS One 2015; 10: e0123097

[30] Faridi KF, Lupton JR, Martin SS et al. Vitamin D deficiency and non-lipid biomarkers of cardiovascular risk. Arch Med Sci 2017; 13: 732-737

[31] Sipahi S, Acikgoz SB, Genc AB et al. The association of vitamin d status and vitamin $\mathrm{d}$ replacement therapy with glycemic control, serum uric acid levels, and microalbuminuria in patients with type 2 diabetes and chronic kidney disease. Med Princ Pract 2017; 26: 146-151 
[32] Vallianou N, Bountziouka V, Akalestos T et al. Vitamin D status and health correlates among apparently healthy participants in an urban, sunny region. Cent Eur J Public Health 2012; 20: 262-269

[33] Wells G, Shea B, O'Connell D et al. The Newcastle-Ottawa Scale (NOS) for assessing the quality of nonrandomised studies in meta-analyses. In: http://www.ohri.ca/programs/clinical_epidemiology/oxford.asp; 2000

[34] Modesti PA, Reboldi G, Cappuccio FP et al. Panethnic differences in blood pressure in Europe: A systematic review and meta-analysis. PLoS One 2016; 11: e0147601

[35] Higgins JPTSJ, Savović J, Page MJ et al. A revised tool for assessing risk of bias in randomized trials. In: Chandler JMJ, Boutron I, Welch V, Eds. Cochrane Methods. 2016 Wiley; 2016

[36] Sterne JAC, Hernán MA, Reeves BC et al. ROBINS-I: A tool for assessing risk of bias in non-randomised studies of interventions. BMJ 2016; 355 : i4919

[37] Deeks J], Altman DG, Bradburn MJ. Statistical Methods for Examining Heterogeneity and Combining Results from Several Studies in Meta-Analysis. In: Egger M, Davey-Smith G, Altman D, Eds. Systematic Reviews in Health Care. London: Wiley; 2008

[38] Thompson SG. Why and how Sources of Heterogeneity Should be Investigated. In: Egger M, Davey-Smith G, Altman D, Eds. Systematic Reviews in Health Care. London: Wiley; 2008

[39] Sterne JAC, Egger M, Smith GD. Investigating and Dealing with Publication and Other Biases. In: Egger M, Davey-Smith G, Altman D, Eds. Systematic Reviews in Health Care. London: Wiley; 2008

[40] Chaimani A, Higgins JPT, Mavridis D et al. Graphical tools for network meta-analysis in STATA. PLoS One 2013; 8: e76654

[41] Rouse B, Chaimani A, Li T. Network meta-analysis: An introduction for clinicians. Intern Emerg Med 2017; 12: 103-111

[42] Higgins JPT, Jackson D, Barrett JK et al. Consistency and inconsistency in network meta-analysis: Concepts and models for multi-arm studies. Res Synth Meth 2012; 3: 98-110

[43] Sterne JA, Egger M. Funnel plots for detecting bias in meta-analysis: Guidelines on choice of axis. J Clin Epidemiol 2001; 54: 1046-1055

[44] Bakirdögen S, Eren N, Bek SG et al. The association between serum uric acid and 25-hydroxyvitamin $\mathrm{D}$ in peritoneal dialysis patients. Turk Nephrol Dial Transplant J 2018; 27: 162-165

[45] Barcelo A, Esquinas C, Pierola J et al. Vitamin D status and parathyroid hormone levels in patients with obstructive sleep apnea. Respiration 2013; 86: 295-301

[46] Barker T, Henriksen VT, Rogers VE et al. Vitamin D deficiency associates with $y$-tocopherol and quadriceps weakness but not inflammatory cytokines in subjects with knee osteoarthritis. Redox Biol 2014; 2: 466-474

[47] Bener A, Al-Hamaq AO, Kurtulus EM et al. The role of vitamin D, obesity and physical exercise in regulation of glycemia in type 2 diabetes mellitus patients. Diabetes Metab Syndr 2016; 10: 198-204

[48] Chan HL, Elkhashab M, Trinh H et al. Association of baseline vitamin D levels with clinical parameters and treatment outcomes in chronic hepatitis B. J Hepatol 2015; 63: 1086-1092

[49] Chen X, Wu W, Wang L et al. Association between 25-Hydroxyvitamin $\mathrm{D}$ and epicardial adipose tissue in Chinese non-obese patients with type 2 diabetes. Med Sci Monit 2017; 23: 4304-4311

[50] Jalali G, Hami M, Namaee $\mathrm{N}$ et al. Relationship between vitamin D deficiency and metabolic syndrome in renal transplant patients in Mashhad, Iran. Shiraz E Med J 2017; 18: e40985

[51] Kim WT, Kim Y], Yun S] et al. Role of 1,25-dihydroxy vitamin D3 and parathyroid hormone in urinary calcium excretion in calcium stone formers. Yonsei Med J 2014; 55: 1326-1332
[52] Myrda K, Rozentryt P, Niedziela JT et al. Clinical and laboratory determinants of low serum level of 25 -hydroxyvitamin $D$ during escalation of pharmacotherapy in heart failure patients. Kardiochir Torakochirurgia Pol 2015; 12: 216-227

[53] Nardin M, Verdoia M, Schaffer A et al. Vitamin D status, diabetes mellitus and coronary artery disease in patients undergoing coronary angiography. Atherosclerosis 2016; 250: 114-121

[54] Parveen S, Zeeshan R, Sultan S et al. Serum 25-hydroxyvitamin D Insufficiency in B-Chronic Lymphoid Leukemia at the Time of Disease Presentation in Pakistan. Asian Pac J Cancer Prev 2015; 16: 5983-5986

[55] Shrestha S, Shrestha L, Jha DK. Vitamin D status among individuals attended in Health Home Care Centre, Lalitpur, Nepal. Nepal Med Coll J 2012; 14: 84-87

[56] Uzunova I, Kirilov G, Zacharieva S et al. Does Vitamin D Status Correlate with Cardiometabolic Risk Factors in Adults with Growth Hormone Deficiency? Horm Metab Res 2017; 49: 499-506

[57] Wang Y, Zhang H. Serum 25-Hydroxyvitamin D3 Levels Are Associated with Carotid Intima-Media Thickness and Carotid Atherosclerotic Plaque in Type 2 Diabetic Patients. J Diabetes Res 2017; 3510275

[58] Xiao X, Wang Y, Hou Y et al. Vitamin D deficiency and related risk factors in patients with diabetic nephropathy. J Int Med Res 2016; 44: 673-684

[59] Yilmaz H, Kaya M, Sahin M et al. Is vitamin D status a predictor glycaemic regulation and cardiac complication in type 2 diabetes mellitus patients? Diabetes Metab Syndr 2012; 6: 28-31

[60] Chin KY, Nirwana SI, Ngah WZ. Significant association between parathyroid hormone and uric acid level in men. Clin Interv Aging 2015; 10: 1377-1380

[61] Hernandez JL, Nan D, Martinez J et al. Serum uric acid is associated with quantitative ultrasound parameters in men: Data from the Camargo cohort. Osteoporos Int 2015; 26: 1989-1995

[62] Pirro M, Mannarino MR, Bianconi V et al. Uric acid and bone mineral density in postmenopausal osteoporotic women: the link lies within the fat. Osteoporos Int 2017; 28: 973-981

[63] Sakoh T, Nakayama M, Tsuchihashi T et al. Associations of fibroblast growth factor 23 with urate metabolism in patients with chronic kidney disease. Metabolism 2016; 65: 1498-1507

[64] Şengül E, Binnetoğlu E, Yilmaz A. The association between uric acid and vitamin $D$ levels in patients with chronic kidney disease. Turk Nephrol Dial Transplant J 2011; 20: 163-167

[65] Veronese N, Bolzetta F, De Rui M et al. Serum uric acid and incident osteoporotic fractures in old people: The PRO.V.A study. Bone 2015; 79: 183-189

[66] Chen W, Roncal-Jimenez C, Lanaspa M et al. Uric acid suppresses 1 alpha hydroxylase in vitro and in vivo. Metab-Clin Exp 2014; 63: 150-160

[67] Arguelles LM, Langman CB, Ariza AJ et al. Heritability and environmental factors affecting vitamin $D$ status in rural Chinese adolescent twins. J Clin Endocrinol Metab 2009; 94: 3273-3281

[68] Köttgen A, Albrecht E, Teumer A et al. Genome-wide association analyses identify 18 new loci associated with serum urate concentrations. Nat Genet 2013; 45: 145-154

[69] Nath SD, Voruganti VS, Arar NH et al. Genome scan for determinants of serum uric acid variability. J Am Soc Nephrol 2007; 18: 3156-3163

[70] Wjst M, Altmüller ], Braig $C$ et al. A genome-wide linkage scan for 25-OH-D(3) and 1,25-(OH)2-D3 serum levels in asthma families. J Steroid Biochem Mol Biol 2007; 103: 799-802

[71] Yang B, Mo Z, Wu C et al. A genome-wide association study identifies common variants influencing serum uric acid concentrations in a Chinese population. BMC Med Genomics 2014; 7: 10 
[72] Cheung CL, Lau KS, Sham PC et al. Genetic variant in vitamin D binding protein is associated with serum 25-hydroxyvitamin $D$ and vitamin $D$ insufficiency in southern Chinese. J Hum Genet 2013; 58: 749-751

[73] Zhang Z, He JW, Fu WZ et al. An analysis of the association between the vitamin D pathway and serum 25-hydroxyvitamin D levels in a healthy Chinese population. J Bone Miner Res 2013; 28: 1784-1792

[74] Thakkinstian A, Anothaisintawee T, Chailurkit L et al. Potential causal associations between vitamin $\mathrm{D}$ and uric acid: Bidirectional mediation analysis. Sci Rep 2015; 5: 14528

[75] Hayashino Y, Okamura S, Tsujii S et al. Association of serum uric acid levels with the risk of development or progression of albuminuria among Japanese patients with type 2 diabetes: A prospective cohort study [Diabetes Distress and Care Registry at Tenri (DDCRT 10)]. Acta Diabetol 2016; 53: 599-607
[76] Sanchis-Gomar F, Salvagno GL, Lippi G. Inhibition of xanthine oxidase and exercise on serum uric acid, 25(OH)D3, and calcium concentrations. Clin Lab 2014; 60: 1409-1411

[77] Saleh F, Jorde R, Sundsfjord J et al. Causes of secondary hyperparathyroidism in a healthy population: The Tromso study. J Bone Miner Metab 2006; 24: 58-64

[78] Sugimoto R, Watanabe H, Ikegami K et al. Down-regulation of ABCG2, a urate exporter, by parathyroid hormone enhances urate accumulation in secondary hyperparathyroidism. Kidney Int 2017; 91: 658-670 